\title{
Gravitating Fermions in an Infinite Configuration Space`
}

\author{
A. Pflug
}

Institut für Theoretische Physik, Universität Wien, Boltzmanngasse 5, A-1090 Wien, Austria

\begin{abstract}
For a system of (infinitely many) nonrelativistic gravitating fermions described rigorously by Thomas-Fermi theory, a nontrivial limit of infinite configuration volume $|\Lambda|$ is shown to exist for the microcanonical free energy, and for the entropy divided by $\log |\Lambda|$. It can be calculated explicitly using the scaling behaviour of the (ground state). Thomas-Fermi equation and shows a phase transition at zero energy. For all (possible) negative energies, the heat capacity of the infinitely extended system is negative and a nonzero fraction of the particles is in the condensed phase.
\end{abstract}

\section{Introduction}

For a system of $N$ nonrelativistic gravitating fermions, a nontrivial limit $N \rightarrow \infty$ exists for the entropy (see [4]), the free energy [5] and the thermodynamical pressure [8], if those functions, together with their arguments, are appropriately scaled with $N$ (see [4]). For nonzero temperatures, the system has to be enclosed in a "box" = bounded open region $\Lambda \subseteq \mathbb{R}^{3}$ whose linear dimensions have to shrink proportional to $N^{-1 / 3}$ in order to give a nontrivial limit for the collapsing system. To work with an $N$-independent confining region (which is conceptually simpler) we choose an $N$-dependent length scale and replace the original Hamiltonian [with units $h=(4 \pi)^{1 / 3}, m=1 / 2$, gravitational constant $\kappa=1 / \pi$ ]

$$
H_{N, \Lambda}=2^{-2 / 3} \pi^{-4 / 3} \sum_{i=1}^{N}\left(-\Delta_{i}\right)-\frac{1}{4 \pi} \sum_{i<j}\left|x_{i}-x_{j}\right|^{-1}
$$

by

$$
\begin{aligned}
H_{N, \Lambda}^{\sim}= & N^{-4 / 3} U^{\dagger} H_{N, \Lambda_{N}} U=N^{-2 / 3} 2^{-2 / 3} \pi^{-4 / 3} \sum_{i=1}^{N}\left(-\Delta_{i}\right)-\frac{1}{4 \pi N} \sum_{i<j}\left|x_{i}-x_{j}\right|^{-1} \\
& \left(U^{\dagger} x \uparrow \Lambda_{N} U=N^{-1 / 3} x \uparrow \Lambda\right),
\end{aligned}
$$

* Work supported in part by Fonds zur Förderung der wissenschaftlichen Forschung in Österreich, Project No. 3569 
where $\Lambda_{N}$ is the contraction (by a factor $N^{-1 / 3}$ in linear dimensions) of the bounded open region $\Lambda$ and $\Delta_{i}$ the $i$-th particle Laplacian with Dirichlet boundary conditions. Assuming a single kind of electrically neutral, spinless fermions, we restrict $H_{N, \Lambda}$ and $H_{N, \Lambda}^{\sim}$ to $\mathscr{H}_{N}(\Lambda)$, the totally antisymmetric $N$-fold tensor product of $\mathscr{L}^{2}(\Lambda)$; in this case, the ground state energy of $H_{N, \Lambda_{N}}$ is proportional to $N^{7 / 3}$ and $H_{N, \Lambda}^{\sim}$ is therefore stable in the usual sense [9].

If $s$ is a nonnegative real number and $\left(\Omega_{N}\right)_{N \in \mathbb{N}}$ a sequence of natural numbers with $\lim _{N \rightarrow \infty} N^{-1} \log \Omega_{N}=S$, then a nontrivial limit

$$
\lim _{N \rightarrow \infty} N^{-7 / 3} E\left(N, \Omega_{N}, \Lambda_{N}\right)=\lim _{N \rightarrow \infty} N^{-1} E^{\sim}\left(N, \Omega_{N}, \Lambda\right):=\varepsilon(s, \Lambda)
$$

is shown to exist [4], where the mean energy $E^{(\sim)}(N, \Omega, \Lambda)$ is given by $\Omega^{-1} \sum_{i=1}^{\Omega} E_{i}^{(\sim)}$ $\left(E_{i}^{(\sim)}\right.$ are the ordered eigenvalues of $\left.H_{N, \Lambda}^{(\sim)}\right)$. As $s \rightarrow \varepsilon(s, \Lambda)$ is a strictly increasing continuous function, it can be inverted to give $s\left(\varepsilon\left(s^{\prime}, \Lambda\right), \Lambda\right)=s^{\prime}$ where

$$
s(\varepsilon, \Lambda)=\lim _{N \rightarrow \infty} N^{-1} S\left(\varepsilon N^{7 / 3}, H_{N, \Lambda_{N}}\right)=\lim _{N \rightarrow \infty} N^{-1} S\left(\varepsilon N, H_{N, \Lambda}^{\sim}\right)
$$

with $S(E, H)=\log \operatorname{Tr} \Theta(E-H)$. Analogously,

$$
\lim _{N \rightarrow \infty} N^{-7 / 3} F\left(N, T N^{4 / 3}, \Lambda_{N}\right)=\lim _{N \rightarrow \infty} N^{-1} F^{\sim}(N, T, \Lambda)=f(T, \Lambda),
$$

where

$$
F^{(\sim)}(N, T, \Lambda)=-T \log \operatorname{Tr} \exp \left(-\frac{1}{T} H_{N, \Lambda}^{(\sim)}\right)
$$

\section{Notations and Definitions}

As shown in [4], $\varepsilon(s, \Lambda)$ respectively $f(T, \Lambda)$ are given by the (global) minimum value of the functionals $\hat{\varepsilon}_{s}(\varrho)$ respectively $\hat{f}_{T}(\varrho)$ over an appropriate space $\mathscr{D}(\Lambda)$ of nonnegative functions $\varrho: \Lambda \rightarrow \mathbb{R}$ with $\int \varrho(x) d^{3} x=1$ (see Sect. 2). $\hat{\varepsilon}_{s}(\varrho):=\hat{\varepsilon}(\hat{T}(s, \varrho), \varrho)$ : $=\hat{\tau}(\hat{T}(s, \varrho), \varrho)+\hat{w}(\varrho)$ where $\hat{\tau}(T, \varrho):=\int_{\Lambda}^{\Lambda} \theta(T,-\mu(T, \varrho(x))) d^{3} x$ is the (mean) energy per particle of a free fermi gas at temperature $T$ with prescribed spatial density distribution $\varrho(x)$ (in a suitable thermodynamic limit)

$$
\begin{aligned}
\theta(T, a) & :=\int_{0}^{\infty} p^{4}\left(1+\exp \left(\frac{1}{T}\left(p^{2}+a\right)\right)\right)^{-1} d p \\
\theta(0, a) & :=\frac{1}{5}|a|^{5 / 2} \Theta(-a) \\
v(T, a) & :=\int_{0}^{\infty} p^{2}\left(1+\exp \left(\frac{1}{T}\left(p^{2}+a\right)\right)\right)^{-1} d p \\
v(0, a): & =\frac{1}{3}|a|^{3 / 2} \Theta(-a) .
\end{aligned}
$$

$\hat{T}(s, \varrho)$ respectively $\mu(T, v)$ are uniquely defined by $\hat{s}(\hat{T}(s, \varrho), \varrho)=s$ and $v\left(T,-\mu\left(T, v^{\prime}\right)\right)=v^{\prime}$ where $\hat{s}(T, \varrho):=\int_{\Lambda} \sigma(T, \varrho(x)) d^{3} x$ and $\sigma(T, v)$ is the volume density 
of entropy for a free fermi gas with temperature $T$ and density $v$. The potential energy functional $\hat{w}(\varrho)$ is given by $\hat{w}(\varrho):=\frac{1}{2} \int_{\Lambda} \hat{v}(\varrho, x) \varrho(x) d^{3} x$ with $\hat{v}(\varrho, x)$ :

$=-\frac{1}{4 \pi} \int_{\Lambda} \varrho(y)|x-y|^{-1} d^{3} y$.

In the sequel, we shall need the quantities $\hat{u}(T, \varrho):=\sup _{x} \mu(T, \varrho(x))$,

$$
\hat{\mu}(T, \varrho):=\sup _{x}(\mu(T, \varrho(x))+\hat{v}(\varrho, x)) .
$$

A necessary condition for $\varrho \in \mathscr{D}(\Lambda)$ to minimize $\hat{\varepsilon}_{s}(\varrho)$ respectively $\hat{f}_{T}(\varrho)$ : $=\hat{\varepsilon}(T, \varrho)-T \cdot \hat{s}(T, \varrho)$ is the validity of the Thomas-Fermi $(:=$ T.F. $)$ equation which reads $D \hat{\varepsilon}_{s}(\varrho) \equiv 0$ respectively $D \hat{f}_{T}(\varrho) \equiv 0, D$ being the Gâteaux functional derivative. Both T.F. equations can be written in the form

$$
\mu(T, \varrho(x))=\hat{\mu}(T, \varrho)-\hat{v}(\varrho, x)
$$

for all $x \in \Lambda$, with $T \equiv \hat{T}(s, \varrho)$ in the microcanonical case.

We denote by $\mathscr{E}^{\prime}(s, \Lambda)$ respectively $\mathscr{F}^{\prime}(T, \Lambda)$ the set of all solutions $\varrho$ of $(1)$ which lie in $\mathscr{D}(\Lambda) . \mathscr{E}(s, \Lambda),(\mathscr{F}(T, \Lambda))$ is the set of all $\varrho \in \mathscr{D}(\Lambda)$ which minimize $\hat{\varepsilon}_{s},\left(\hat{f}_{T}\right)$ globally.

\section{Properties of the (Microcanonical) Variational Principle}

The functional $\hat{T}(s, \varrho)$ is well defined and $<\infty$ for all $\varrho \in \mathscr{D}(\Lambda):=\left\{0 \leqq \varrho \in \mathscr{L}^{1}(\Lambda) \mid \int_{\Lambda} \varrho(x) d^{3} x=1\right\}$ because there is a relation

$$
[3|\Lambda|(6+a)+s]^{-1} s \frac{d}{d s} k_{F}\left(\frac{s}{2}\right)<\frac{d}{d s} \hat{\tau}_{s}(\varrho)=\hat{T}(s, \varrho)<5 c^{2 / 3}\left(\exp \left(\frac{4}{3} s\right)+4\right):=a
$$

which can be shown with the help of the inequalities (A1.3) and (A2.2)-(A2.5) in the appendix $\left(\hat{\tau}_{s}(\varrho):=\hat{\tau}(\hat{T}(s, \varrho), \varrho)\right)$.

Here, $|\Lambda|:=\int_{\Lambda} d^{3} x, \tau_{F}(s, v)=v^{2 / 3} k_{F}(s)$ is the energy per particle of a free fermi gas (with entropy per particle $=s$ and density $=v$ ) and $0<c<\infty$ is a positive real number with $\int_{\Lambda} \varrho(x) \Theta(c-\varrho(x)) d^{3} x \geqq 1 / 2$ which exists for every $\varrho \in \mathscr{D}(\Lambda)$. [If $\varrho \in \mathscr{D}^{p}(\Lambda):=\mathscr{D}(\Lambda) \cap \mathscr{L}^{p}(\Lambda), \quad p>1$, we can choose $c=2^{1 /(p-1)}\|\varrho\|_{p}^{p /(p-1)}$, but $\sup \{\hat{T}(s, \varrho) \mid \varrho \in \mathscr{D}(\Lambda)\}=+\infty$ for all $s>0$.]

As $\hat{s}(T, \varrho) \leqq|\Lambda| \sigma\left(T,|\Lambda|^{-1}\right)<\infty, \hat{s}(T, \varrho)$ is uniformly bounded on $\mathscr{D}(\Lambda)$ for every fixed $T \geqq 0$. The nonnegative functionals $\hat{\tau}(\varrho)$ respectively $-\hat{w}(\varrho)$ can be defined on the whole set $\mathscr{D}(\Lambda)$, but as they are simultaneously $+\infty$ on some points, $\hat{\varepsilon}_{s}(\varrho)$ $=\hat{\tau}(\hat{T}(s, \varrho), \varrho)+\hat{w}(\varrho)$ is not everywhere well defined. If we restrict $\hat{\varepsilon}_{s}(\varrho)$ to $\mathscr{D}^{p}(\Lambda)$ with $p>3 / 2, \hat{w}(\varrho)$ is everywhere $>-\infty$ and $\hat{\varepsilon}_{s}(\varrho)$ therefore unique. Choosing $p=5 / 3$, we get the bound

$$
-1<\|\varrho\|_{5 / 3}^{5 / 3}-\|\varrho\|_{5 / 3}^{5 / 6}<\hat{\varepsilon}_{s}(\varrho)<35\|\varrho\|_{5 / 3}^{5 / 3}\left(\exp \left(\frac{4}{3} s\right)+5\right)
$$


where we have used (2), (A2.5), and (A2.2) in Appendix 2 and [7]. Minimizing the right hand side over $\mathscr{D}(\Lambda)$ gives the inequality

$$
\|\bar{\varrho}\|_{5 / 3}^{5 / 6}<1+35|\Lambda|^{-2 / 3}\left(\exp \left(\frac{4}{3} s\right)+5\right)
$$

for every minimizing density $\bar{\varrho}$. This allows to restrict the variational principle to a subset of $\mathscr{D}^{5 / 3}(\Lambda)$ bounded in the $\mathscr{L}^{5 / 3}(\Lambda)$-norm as given by (4). The Gâteaux functional derivative exists on a norm dense subset of $\mathscr{D}^{5 / 3}(\Lambda)$ [containing all functions $\varrho(x) \geqq \delta>0$ almost everywhere on $\Lambda$ with arbitrary $\delta]$ and $\left[D \hat{\varepsilon}_{s}(\varrho)\right]\left(\varrho^{\prime}-\varrho\right)$ can be defined for arbitrary $\varrho, \varrho^{\prime} \varepsilon \in \mathscr{D}^{5 / 3}(\Lambda)$, but may be $-\infty$. Therefore $D \hat{\varepsilon}_{s}(\varrho)$ has to be the zero functional at every (local or global) minimum of $\varrho \rightarrow \hat{\varepsilon}_{s}(\varrho)$ which gives the Thomas-Fermi equation (1).

As the functional $\varrho \rightarrow \hat{\tau}_{s}(\varrho)$ is convex [because it is the minimum of the jointly convex functional $(\sigma, \varrho) \rightarrow \int_{\Lambda} \varrho^{5 / 3}(x) k_{F}(\sigma(x) / \varrho(x)) d^{3} x$ over the convex set of functions $\left.\left\{0 \leqq \sigma(x) \mid \int_{\Lambda} \sigma(x) d^{3} x=s\right\}\right], \varrho \rightarrow \tau_{s}(\varrho)$ is lower semicontinuous in the weak $\mathscr{L}^{5 / 3}(\Lambda)$ topology on $\mathscr{D}^{5 / 3}(\Lambda)$ (because $|\Lambda|<\infty$, see [1], p. 263), but not continuous. If the sequence $\left(\varrho_{n}\right)_{n \in \mathbb{N}} \leqq \mathscr{D}^{5 / 3}(\Lambda)$ converges to $\varrho$ weakly in $\mathscr{L}^{5 / 3}(\Lambda)$, then $\varrho \in \mathscr{D}^{5 / 3}(\Lambda)$ and $\sup _{n \in \mathrm{N}}\left\|\varrho_{n}\right\|_{5 / 3} \leqq c<\infty$ by uniform boundedness. $\left|\hat{w}(\varrho)-\hat{w}\left(\varrho_{n}\right)\right| \leqq c\left\|V_{A} *\left(\varrho-\varrho_{n}\right)\right\|_{5 / 2}$ where * means convolution and $V_{\Lambda}(x):=|x|^{-1} \Theta(d(\Lambda)-|x|)$ with $d(\Lambda):=$ sup $\{\mid x-y \| x, y \in \Lambda\}$. As $y \rightarrow|x-y|^{-1} \Theta(d(\Lambda)-|x-y|)$ is in $\mathscr{L}^{5 / 2}\left(\mathbb{R}^{3}\right)$ for every $x \in \mathbb{R}^{3}$, $\left[V_{A} *\left(\varrho-\varrho_{n}\right)\right](x)$ converges pointwise to zero. By dominated convergence (which follows from $\left\|V_{\Lambda} *\left(\varrho-\varrho_{n}\right)\right\|_{\infty} \leqq 2 c\left\|V_{\Lambda}\right\|_{5 / 2}$ because $\left.|\Lambda|<\infty\right), \lim _{n \rightarrow \infty}\left\|V_{\Lambda} *\left(\varrho-\varrho_{n}\right)\right\|_{5 / 2}=0$ and $\varrho \rightarrow \hat{w}(\varrho)$ is continuous in the weak $\mathscr{L}^{5 / 3}(\Lambda)$ topology. Therefore $\hat{\varepsilon}_{s}(\varrho)$ is lower semicontinuous in this topology and reaches its infimum on $\mathscr{D}^{5 / 3}(\Lambda)$, because every subset of $\mathscr{D}^{5 / 3}(\Lambda)$ bounded in the $\mathscr{L}^{5 / 3}(\Lambda)$-norm is compact in the weak $\mathscr{L}^{5 / 3}(\Lambda)$ topology, $\mathscr{L}^{5 / 3}(\Lambda)$ being reflexive. Consequently $\mathscr{E}(s, \Lambda) \neq \emptyset$ for all $s \geqq 0$ and all bounded open regions $\Lambda \subseteq \mathbb{R}^{3}$. The same is true for $\mathscr{F}(T, \Lambda)$ as $\varrho \rightarrow-T \cdot \hat{s}(T, \varrho)$ is convex (and bounded) on $\mathscr{D}(\Lambda)$ for fixed $T$. From the convexity of $s \rightarrow \hat{\tau}_{s}(\varrho)$ together with (2) we conclude that $s \rightarrow \varepsilon(s, \Lambda)$ is continuous and strictly increasing, so that the inverse function $\varepsilon \rightarrow s(\varepsilon, \Lambda)$ exists on the closed half-line $\left[\varepsilon^{*}(\Lambda), \infty\right)$ where $\varepsilon^{*}(\Lambda):=\varepsilon(0, \Lambda)$. [We shall see later on that $\varepsilon^{*}(\Lambda)=\varepsilon^{*}=$ const independent of $\Lambda$, if $\Lambda$ contains a sphere $S_{R^{*}}$ with radius $R^{*}$.] As an easy consequence of the above discussion, we get

Lemma 1. If a sequence $\left(\varrho_{n}\right)_{n \in \mathbb{N}}$ with $\varrho_{n} \in \mathscr{E}\left(s_{n}, \Lambda\right)$ converges to $\varrho$ in the weak $\mathscr{L}^{5 / 3}(\Lambda)$ topology and if $s_{n} \leqq c<\infty$ for all $n \in \mathbb{N}$, then $\lim _{n \rightarrow \infty} s_{n}=s$ exists, $\varrho \in \mathscr{E}(s, \Lambda)$ and $\varepsilon(s, \Lambda)=\lim _{n \rightarrow \infty} \hat{\varepsilon}_{s}\left(\varrho_{n}\right)=\lim _{n \rightarrow \infty} \varepsilon\left(s_{n}, \Lambda\right)$.

As, for sufficiently large $\Lambda$, the function $s \rightarrow \varepsilon(s, \Lambda)$ is not convex on the whole domain, the existence of a microcanonical temperature $T(s, \Lambda):=\frac{d}{d s} \varepsilon(s, \Lambda)$ is not clear a priori; therefore we introduce a generalized version of this notion in 
Lemma 2. If we define

$$
\begin{aligned}
T_{+(-)}^{r}(s, \Lambda) & :=\lim \sup _{0<d \rightarrow 0} \text { (inf) } \frac{1}{d}(\varepsilon(s+d, \Lambda)-\varepsilon(s, \Lambda)) \\
T_{+(-)}^{l}(s, \Lambda) & :=\lim \sup _{0<d \rightarrow 0}(\inf ) \frac{1}{d}(\varepsilon(s, \Lambda)-\varepsilon(s-d, \Lambda)) \\
T_{+}(s, \Lambda) & :=T_{-}^{r}(s, \Lambda), \quad T_{-}(s, \Lambda):=T_{+}^{l}(s, \Lambda), \\
T_{+(-)}^{\prime}(s, \Lambda): & =\sup (\inf )\{\hat{T}(s, \varrho) \mid \varrho \in \mathscr{E}(s, \Lambda)\}, \\
T_{+(-)}^{\prime \prime}(s, \Lambda) & =\lim \inf _{0<d \rightarrow 0}(\sup ) T_{+(-)}^{\prime}(s( \pm) d, \Lambda)
\end{aligned}
$$

then every accumulation point of $d_{n}^{-1}\left(\varepsilon\left(s+d_{n}, \Lambda\right)-\varepsilon(s, \Lambda)\right), d_{n} \neq 0, \lim _{n \rightarrow \infty} d_{n}=0$ lies in the closed interval $I(s, \Lambda):=\left[T_{-}^{r}(s, \Lambda), T_{+}^{l}(s, \Lambda)\right]$ and

$$
T_{+}^{\prime \prime}(s, \Lambda) \leqq T_{-}^{r}(s, \Lambda) \leqq T_{+}^{r}(s, \Lambda) \leqq T_{-}^{\prime}(s, \Lambda) \leqq T_{+}^{\prime}(s, \Lambda) \leqq T_{-}^{l}(s, \Lambda) \leqq T_{+}^{l}(s, \Lambda) \leqq T_{-}^{\prime \prime}(s, \Lambda) .
$$

The easy proof follows from the variational principle and a uniform estimate of difference quotients.

Remark 1. If $\varepsilon(s, \Lambda)$ is convex in a neighborhood of a point $s_{0}$, then it is differentiable there. If $\mathscr{E}\left(s_{0}, \Lambda\right)$ consists of a single point and $\varepsilon(s, \Lambda)$ is concave in a neighborhood of $s_{0}$, then it can be shown that the derivative at $s_{0}$ exists, using special properties of the solutions of the T.F. equation (1) (see [2]).

\section{The Main Result}

It has been shown numerically in [6], that for a sufficient large, spherical $\Lambda \cong \mathbb{R}^{3}$, the function $\varepsilon \rightarrow s(\varepsilon, \Lambda)$ is no longer concave on the whole domain $\left[\varepsilon^{*}, \infty\right)$, but there is no first-order phase transition in the microcanonical picture. The latter only occurs in the canonical case where the function $T \rightarrow f(T, \Lambda)$ is not differentiable for a certain critical $T=T_{c}(R)$ depending on the radius $R$ of the spherical "box" $S_{R}$. Although the existence of this box is an unphysical assumption (but necessary to guarantee the existence of all partition functions because the gravitational force alone, vanishing in the limit of infinite interparticle distance, would not prevent the complete evaporation of a star at nonzero temperature) we try to get rid of its influence by taking after all other limits leading to the T.F. theory, the limit $\Lambda \rightarrow \mathbb{R}^{3}$. This limit exists for the microcanonical free energy $f_{m}^{ \pm}(\varepsilon, \Lambda)=\varepsilon-T_{ \pm}(s(\varepsilon, \Lambda), \Lambda) s(\varepsilon, \Lambda)$ and for the "rescaled" entropy $(\log |\Lambda|)^{-1} s(\varepsilon, \Lambda)$ and shows a phase transition in the microcanonical description of the spatially infinite system at $\varepsilon=0$ which corresponds to the beginning of the formation of a condensed phase in cold gas cloud, i.e. the birth of a star. We state our results quantitatively in the

Theorem. Let $\left(\Lambda_{n}\right)_{n \in \mathbb{N}}$ be a sequence of bounded open regions $\subseteq \mathbb{R}^{3}$ with $\lim _{n \rightarrow \infty}\left|\Lambda_{n}\right|=+\infty$ and $\lim _{n \rightarrow \infty}\left(\log R_{+}\left(\Lambda_{n}\right)\right)^{-1} \log R_{-}\left(\Lambda_{n}\right)=1$ [where $R_{+(-)}(\Lambda)$ is the 
radius of the smallest (greatest) sphere containing (contained in) $\Lambda$ ], then
i) $\limsup _{|\Lambda| \rightarrow \infty}(\log |\Lambda|)^{-1} s(\varepsilon, \Lambda) \leqq 1, \varepsilon^{*} \leqq \varepsilon<\infty$;
ii) $\lim _{n \rightarrow \infty}\left(\log \left|\Lambda_{n}\right|\right)^{-1} s\left(\varepsilon, \Lambda_{n}\right)=1-\left(\varepsilon / \varepsilon^{*}\right)^{3 / 7} \Theta(-\varepsilon), \varepsilon^{*} \leqq \varepsilon<\infty$;
iii) $\lim _{R \rightarrow \infty} f_{m}^{ \pm}\left(\varepsilon, S_{R}\right)=\frac{1}{3} \varepsilon^{*}\left(7\left(\varepsilon / \varepsilon^{*}\right)^{4 / 7}-4 \varepsilon / \varepsilon^{*}\right), \varepsilon^{*} \leqq \varepsilon<0$, $\lim _{|\Lambda| \rightarrow \infty} f_{m}^{ \pm}(\varepsilon, \Lambda)=-\infty, 0<\varepsilon<\infty ;$
iv) $\lim _{|\Lambda| \rightarrow \infty}\left\{\hat{\varepsilon}(T, \varrho) \mid \varrho \in \mathscr{F}^{\prime}(T, \Lambda)\right\}=\frac{3}{2} T, 0<T<\infty$;
v) $\lim _{|\Lambda| \rightarrow \infty}\left\{T \mid \varrho \in \mathscr{F}^{\prime}(T, \Lambda), \hat{\varepsilon}(T, \varrho)=\varepsilon\right\}=\frac{2}{3} \varepsilon \Theta(\varepsilon), \varepsilon^{*} \leqq \varepsilon<\infty$;
vi) $\lim _{R \rightarrow \infty}\left\{T \log \mid S_{R} \| \varrho \in \mathscr{F}\left(T, S_{R}\right), \hat{\varepsilon}(T, \varrho)=\varepsilon\right\}=\left(-\frac{7}{3} \varepsilon^{*}\right)\left(\varepsilon / \varepsilon^{*}\right)^{4 / 7}$
$=\lim _{R \rightarrow \infty}\left\{-\hat{\mu}(T, \varrho) \mid \varrho \in \mathscr{F}\left(T, S_{R}\right), \hat{\varepsilon}(T, \varrho)=\varepsilon\right\}, \varepsilon^{*} \leqq \varepsilon<0$

vii) $\lim _{|\Lambda| \rightarrow \infty}\left\{-\hat{\mu}(T, \varrho)(\log |\Lambda|)^{-1} \mid \varrho \in \mathscr{F}^{\prime}(T, \Lambda)\right\}=T$.

Remark 2. For $\varepsilon=0$, there is no definite volume dependence of $T$ or $\hat{\mu}$ in the limit $\Lambda \rightarrow \mathbb{R}^{3}$ which may be considered as an indication for the microcanonical phase transition [= nondifferentiability of the (rescaled) entropy (ii) as function of energy $=$ nonuniqueness of the (rescaled) temperature (vi)] which occurs at $\varepsilon=0$. Furthermore, the microcanonical free energy is discontinuous there. The limit of the (rescaled) temperature equals the inverse derivative of the (rescaled) entropy for $\varepsilon^{*} \leqq \varepsilon<0$ and $0<\varepsilon<\infty$ being $+\infty$ for $\varepsilon>0$. The canonical free energy $f(T, \Lambda)$ has the trivial limit $\lim _{|\Lambda| \rightarrow \infty}(\log |\Lambda|)^{-1} f(T, \Lambda)=-T$ for all $T \geqq 0$ and the interesting region $\varepsilon \leqq 0$ corresponds to the single point $T=0$. Although, physically, an interstellar gas cloud is not an isolated system, it can only lose energy (by radiation) but not take it from a heath bath, so that the microcanonical description seems closer to reality.

Remark 3. The way $\Lambda_{n}$ is allowed to tend to infinity, is strictly more general than the usually considered van Hove-convergence (see [9]) where surface effects have to vanish. This generality is a consequence of the logarithmic rescaling of the entropy and its monotonicity in $\Lambda$; as this monotonicity is not necessarily valid for the microcanonical temperature, the (nontrivial part of the) limit for the microcanonical free energy is only proven for spherical regions.

Proof. i) It is shown in Appendix 3, that there exists a nonincreasing function $T \rightarrow A(T), 0<T, A(T)<\infty$ such that $\hat{u}(T, \varrho) \leqq 0$ for all $\varrho \in \mathscr{F}^{\prime}(T, \Lambda)$ if $|\Lambda| \geqq A(T)$ and constants $0<b, B<\infty$ with $\|\hat{v}(\varrho, \cdot)\|_{\infty}<b, b>\left|\varepsilon^{*}\right|$ for all $\varrho \in \mathscr{F}^{\prime}(T, \Lambda)$ and all $T \geqq 0$ if $|\Lambda| \geqq B$.

Using the inequalities (A1.3) and (A2.5) in the appendix we obtain the bounds

$$
\begin{aligned}
& \hat{\varepsilon}(3(\varepsilon+b), \varrho)>\varepsilon \\
& \hat{s}(3(\varepsilon+b), \varrho) \leqq 6+\frac{3}{2} \log (\varepsilon+b)+\log |\Lambda|
\end{aligned}
$$


for $\quad \varepsilon^{*} \leqq \varepsilon<\infty \quad$ and $\quad \varrho \in \mathscr{F}^{\prime}(3(\varepsilon+b), \Lambda), \quad$ if $\quad|\Lambda| \geqq A(3(\varepsilon+b))+B$. As $s(\varepsilon, \Lambda)=\sup \left\{\hat{s}(T, \varrho) \mid \varrho \in \mathscr{F}^{\prime}(T, \Lambda), \hat{\varepsilon}(T, \varrho)=\varepsilon\right\}$ this proves i).

ii), iii), and vi) are treated in Sects. 4 and 5. iv) follows from the estimate

$$
\frac{1}{5} T^{3 / 2} \exp \left[-\frac{1}{T}(|u|+b)\right] \leqq \varrho(x) \leqq T^{3 / 2} \exp \left(-\frac{1}{T}|u|\right) \quad \text { for all } \quad x \in \Lambda
$$

and all $\varrho \in \mathscr{F}^{\prime}(T, \Lambda)$ with $u:=\hat{u}(T, \varrho) \leqq 0$ [see (A2.4) in Appendix 2]. This last relation holds for all $\Lambda$ with $|\Lambda| \geqq A(T)$. The normalization condition $\int_{A} \varrho(x) d^{3} x=1$
implies that $\|\varrho\|_{\infty}$ and consequently

$$
\|\hat{v}(\varrho, \cdot)\|_{\infty}<\|\varrho\|_{\infty}^{1 / 3}\|\varrho\|_{1}^{2 / 3}
$$

[see (A3.1) in Appendix 3] converge to zero when $|\Lambda|$ goes to infinity so that in this case $\hat{\varepsilon}(T, \varrho)$ tends to the energy per particle of an ideal fermi gas at temperature $T$ and vanishing density which equals the classical value $\frac{3}{2} T$. To prove v) we may restrict the discussion to $\left\{T \geqq 0 \mid \varrho \in \mathscr{F}^{\prime}(T, \Lambda), \hat{\varepsilon}(T, \varrho)=\varepsilon, \hat{u}(T, \varrho) \leqq u\right\}$ for an arbitrary $u \in \mathbb{R}$, because of the relations

$$
\begin{gathered}
\limsup _{|\Lambda| \rightarrow \infty}\left\{T \mid \varrho \in \mathscr{F}^{\prime}(T, \Lambda), \hat{u}(T, \varrho) \geqq u\right\}=0 \\
\limsup _{|\Lambda| \rightarrow \infty}\left\{\hat{\varepsilon}(T, \varrho) \mid \varrho \in \mathscr{F}^{\prime}(T, \Lambda), \hat{u}(T, \varrho) \geqq u\right\}=0
\end{gathered}
$$

following from (6) [for $u \leqq \hat{u}(T, \varrho) \leqq 0]$ and from (32) [for $\hat{u}(T, \varrho)>0]$.

As $T<3(\varepsilon+b)$ for $|\Lambda| \geqq A(3(\varepsilon+b))+B$, the set of $T$ 's in v) has limit points. If one of them is $>0$, then according to the proof of iv) it equals $\frac{2}{3} \varepsilon$ [because $\|\varrho\|_{\infty}$ and $\|\hat{v}(\varrho, \cdot)\|_{\infty}$ both vanish in the limit $\left.|\Lambda| \rightarrow \infty\right]$. Therefore, all nonzero limit points coincide. If zero is a limit point, then it follows again from (6) that $\|\varrho\|_{\infty}$ and $\|\hat{v}(\varrho, \cdot)\|_{\infty}$ both converge to zero. The inequality

$$
\frac{1}{3} T<\frac{1}{v} \theta(T,-\mu)<4(\mu \Theta(\mu)+T), \quad \mu=\mu(T, v),
$$

[see (A2.5) in Appendix 2] shows that in this case $\varepsilon$ must be zero. vii) is an easy consequence of the inequality (6).

\section{An Upper Bound for the Rescaled Entropy}

In order to derive an upper bound for $\lim _{n \rightarrow \infty} \sup \left(\log \left|\Lambda_{n}\right|\right)^{-1} s\left(\varepsilon, \Lambda_{n}\right)$ we only need to consider spherical regions, because $s$ is an increasing function of $\Lambda$ due to the choice of Dirichlet boundary conditions for the kinetic energy. It has been shown in [2], that $\mathscr{E}\left(s, S_{R}\right)$ only contains spherically symmetric densities. We replace therefore $\mathscr{F}^{\prime}\left(T, S_{R}\right)$ by $\mathscr{F}^{\prime \prime}(T, R)$, the nonvoid set of spherically symmetric solutions of the T.F. equation, which in this case, becomes the ordinary differential equation

$$
\ddot{\chi}(r)=r v\left(T, \frac{1}{r} \chi(r)\right),
$$


where $\varrho(r)=\frac{1}{r} \ddot{\chi}(r) \Theta(R-r), 4 \pi \int_{0}^{R} \varrho(r) r^{2} d r=1, \frac{1}{r} \chi(r)=-\mu(T, \varrho(r))=\hat{v}(\varrho, r)-\hat{\mu}(T, \varrho)$ if $0 \leqq r \leqq R$; the dot means the ordinary derivative $\frac{d}{d r}$. The constraint $\varrho \in \mathscr{L}_{\text {loc }}^{1}$ gives $\chi(0)=0$ so that $\chi(r)$ is uniquely specified by $T$ and $\dot{\chi}(0)=-u=-\hat{u}(T, \varrho)$. It is sufficient to find an upper bound for

$$
g(\varepsilon):=\inf \left\{\limsup _{R \rightarrow \infty}\left(\log \left|S_{R}\right|\right)^{-1} s^{\prime}(u, R) \mid \varepsilon^{\prime}(u)>\varepsilon\right\},
$$

where

$$
s^{\prime}(u, R)=\sup \left\{\hat{s}(T, \varrho) \mid \varrho \in \mathscr{F}^{\prime \prime}(T, R), \hat{u}(T, \varrho)=u\right\}
$$

and

$$
\varepsilon^{\prime}(u)=\liminf _{R \rightarrow \infty}\left(\inf \left\{\hat{\varepsilon}(T, \varrho) \mid \varrho \in \mathscr{F}^{\prime \prime}(T, R), \hat{u}(T, \varrho)=u\right\}\right) .
$$

By calculating explicit bounds for the partial derivatives of the function $v(T, a)$ which is convex decreasing in $1 / T$ and a separately, one gets

$$
\begin{aligned}
& 0 \leqq v(T+d, a)-v(T, a) \leqq d(\sqrt{T+d}+\sqrt{u}) \\
& 0 \leqq v(T, a)-v(T, a+d) \leqq d(\sqrt{T}+\sqrt{u})
\end{aligned}
$$

if $-u \leqq a$ and $0 \leqq T, d, a$.

As $\chi_{T, u}(r)$, the unique solution of (9) with $\chi(0)=0$ and $\dot{\chi}(0)=-u$, is convex in $r$, $\frac{1}{r} \chi_{T, u}(r)$ is increasing in $r$ and consequently $\frac{1}{r} \chi(r) \geqq-u=-\hat{u}\left(T, \frac{1}{r} \ddot{\chi}_{T, u}(r) \Theta(R-r)\right)$. Therefore the estimates (10) give a (uniform) Lipschitz constant for $v\left(T, \frac{1}{r} \chi_{T, u}(r)\right)$. By standard perturbation methods of ordinary differential equations we get the bounds

$$
\left|\varrho_{\alpha, u}^{\prime}(r)-\varrho_{\beta, u}^{\prime}(r)\right| \leqq|\alpha-\beta| u^{3 / 2}\left[\exp \left(u^{1 / 4} r\right)+3\right]
$$

if $0 \leqq \alpha, \beta \leqq 1$ and $u>0$, where

$$
\begin{aligned}
& \varrho_{\alpha, u}^{\prime}(\mathrm{r}):=\frac{1}{r} \ddot{\chi}_{\alpha u, u}(r) \\
& \varrho_{\alpha, u}(r):=\varrho_{\alpha, u}^{\prime}(r) \Theta(R(\alpha, u)-r)
\end{aligned}
$$

for all $r \geqq 0$ and $R(\alpha, u):=\sup \left\{R>0 \mid 4 \pi \int_{0}^{R} \varrho_{\alpha, u}^{\prime}(r) r^{2} d r \leqq 1\right\}$. The prime indicates that $\varrho_{\alpha, u}^{\prime}(r)$ is the infinitely extended solution on $\mathbb{R}^{3}$ not subject to any normalization condition. For notational convenience and dimensional reasons, we have put $T=\alpha u$ and restricted $\alpha$, respectively $u$, to $0 \leqq \alpha \leqq 1$ and $u>0$, because, in the sequel, we will only consider the case $T$ converging to zero at fixed $u>0$ (which corresponds to $\varepsilon^{*} \leqq \varepsilon<0$ ), the remaining cases being already discussed in Sect. 3 .

If $R(\alpha, u)<\infty$, then obviously $\left\|\varrho_{\alpha, u}\right\|_{1}=1$ and $\varrho_{\alpha, u} \in \mathscr{F}^{\prime \prime}(\alpha u, R(\alpha, u))$. If $\left\|\varrho_{\alpha, u}^{\prime}\right\|_{1}$ would be $<\infty$, then $0<\left\|\hat{v}\left(\varrho_{\alpha, u}^{\prime}, \cdot\right)\right\|_{\infty}=-\hat{v}\left(\varrho_{\alpha, u}^{\prime}, 0\right)=c<\infty$ and consequently, $\mu\left(\alpha u, \varrho_{\alpha, u}^{\prime}(r)\right)=u-c-\hat{v}\left(\varrho_{\alpha, u}^{\prime}, r\right)>-c$ which gives, together with inequality (A2.4) of 
Appendix 2 a constant lower bound $0 \leqq \frac{1}{5}(\alpha u)^{3 / 2} \exp \left[-(\alpha u)^{-1} c\right]<\varrho_{\alpha, u}^{\prime}(r)$ for all $r \geqq 0$ contradicting the assumption $\left\|\varrho_{\alpha, u}^{\prime}\right\|_{1}=1$ if $\alpha>0$. Therefore $R(\alpha, u)<\infty$ for all $\alpha, u>0$. To invert $R(\alpha, u)$ as a function of $\alpha$, we first conclude from estimate (11) that there is a $0<c(\alpha, u)<\infty$ with

$$
\left|R^{3}(\alpha, u)-R^{3}(\beta, u)\right| \leqq|\alpha-\beta| c(\alpha, u)
$$

for $|\alpha-\beta| \leqq c^{-1}(\alpha, u)$ [because $v(\alpha u, a)$ and consequently $\varrho_{\alpha, u}(R(\alpha, u))$ are strictly positive for $\alpha>0$ and $\varrho_{\alpha, u}(r)$ is nonincreasing in $r$ ] showing that $\alpha \rightarrow R(\alpha, u)$ is (Hölder) continuous. To calculate $R(0, u)$ we conclude from the T.F. equation for $\alpha=0$

$$
\ddot{\chi}_{0, u}=\frac{r}{3}\left|\frac{1}{r} \chi_{0, u}(r)\right|^{3 / 2} \Theta\left(-\frac{1}{r} \chi_{0, u}(r)\right)
$$

that $\chi_{0, u}$ belongs to the one-parameter family of solutions

$$
\chi_{0, \lambda^{4 / 3} u}(r)=\lambda \chi_{0, u}\left(\lambda^{1 / 3} r\right)
$$

For the corresponding $\varrho_{0, u}^{\prime}(r)=\frac{1}{r} \ddot{\chi}_{0, u}(r)$ which has compact support, there is a unique $\lambda^{*}$ with $\lambda^{*}\left\|\varrho_{0, u}^{\prime}\right\|_{1}=1$. Therefore $\varrho^{*}:=\varrho_{0, u^{*}}$ with $u^{*}=\lambda^{* 4 / 3} u$ is the ground state density, i.e. the unique element of $\mathscr{F}^{\prime \prime}(0, R)$ if $R \geqq R^{*}:=\sup \left\{r>0 \mid \varrho^{*}(r)>0\right\}$. Consequently, $\left\|\varrho_{0, u}^{\prime}\right\|_{1}=\left(u / u^{*}\right)^{3 / 4}$ and $R(0, u)=+\infty$ if $0<u<u^{*}, R(0, u) \leqq R^{*}$ if $u \geqq u^{*}$ and

$$
r(u):=\sup \left\{r>0 \mid \varrho_{0, u}^{\prime}(r)>0\right\}=R^{*}\left(u / u^{*}\right)^{-1 / 4} .
$$

Because $\varrho_{\alpha, u}^{\prime}(r)$ is nonincreasing in $r$ and $\varrho_{0, u}^{\prime}(r)=0$ for $r \geqq r(u)$, we get

$$
\left\|\varrho_{\alpha, u}^{\prime}-\varrho_{0, u}^{\prime}\right\|_{\infty} \leqq \alpha u^{3 / 2} c^{*}
$$

with $c^{*}=\exp \left(u^{* 1 / 4} R^{*}\right)+3$ and, finally

$$
R^{3}(\alpha, u) \geqq\left(5 c^{*}\right)^{-1}\left(\alpha u^{3 / 2}\right)^{-1}\left(1-\left(u / u^{*}\right)^{3 / 4}\right)
$$

for $0<u<u^{*}$. Therefore

$$
\left\|\varrho_{\alpha, u}-\varrho_{0, u}^{\prime}\right\|_{\infty} \leqq \alpha u^{3 / 2} c^{*}
$$

if $0 \leqq \alpha \leqq d_{1}:=\left(5 R^{* 3} c^{*} u^{3 / 2}\right)^{-1}\left(u / u^{*}\right)^{3 / 4}\left(1-\left(u / u^{*}\right)^{3 / 4}\right)$.

Conversely, it is possible to show (by an appropriate lower bound to $\varrho_{\alpha, u}^{\prime}$ ) that $R(\alpha, u) \leqq 2(\alpha u)^{-1 / 2}$ if $\alpha \geqq(5 u)^{-1}$. As the range of the continuous function $\alpha \rightarrow R(\alpha, u)$, $\alpha \in(0, \infty)$ is therefore the open half-line $(0, \infty)$, there exists, for every $R>0$ and every $0<u<u^{*}$ at least one $\alpha>0$ with $R(\alpha, u)=R$. We define $\alpha_{+(-)}(R, u)$ to be the $\sup$ (inf) of $\{\alpha>0 \mid R(\alpha, u)=R\}$ and denote the closed interval $\left[\alpha_{-}(R, u), \alpha_{+}(R, u)\right]$ by $J(R, u)$. Given an energy $\varepsilon$ with $\varepsilon^{*} \leqq \varepsilon<0$, we fix a $u$ with $0<u<u^{*}\left(\varepsilon / \varepsilon^{*}\right)^{4 / 7}$, define $\lambda(u):=\left(u / u^{*}\right)^{3 / 4}$ and choose an (arbitrary, but fixed) $r^{\prime}>r(u)=\lambda^{-1 / 3}(u) R^{*}$.

As $u$ remains unchanged in a great part of the following discussion, we take the freedom to omit it without further comment. From (15) together with

$$
\left|\dot{\chi}_{\alpha, u}(r)-\dot{\chi}_{0, u}(r)\right| \leqq \alpha u\left[\exp \left(u^{1 / 4} r\right)+3\right]
$$


(a standard estimate for ordinary differential equations), it is easy to conclude that there is a $0<d_{2} \leqq d_{1}$ with $\mu_{\alpha}(r):=\mu\left(\alpha u, \varrho_{\alpha, u}^{\prime}(r)\right) \leqq 0$ for all $r \geqq r^{\prime}$ if $0 \leqq \alpha \leqq d_{2}$ because $\dot{\chi}_{0, u}(r(u))=\lambda^{4 / 3}\left(-\mu^{*}\right)=u\left(-\mu^{*} / u^{*}\right)>0$. Here $\mu^{*}:=\hat{\mu}\left(0, \varrho^{*}\right)<0$ is the chemical potential of the ground state solution $\varrho^{*}$ if $R \geqq R^{*}$; this follows from the relation $\dot{\chi}_{0, u}(r(u))=-\hat{v}\left(\varrho_{0, u}^{\prime}, r(u)\right)=-\hat{\mu}\left(0, \varrho_{0, u}^{\prime}\right)$ which is not difficult to prove. From the definition of $u$, we get

$$
\hat{\mu}_{\alpha}-\lambda^{4 / 3} \mu^{*}=v_{\alpha}(0)-v_{0}^{\prime}(0),
$$

where $\hat{\mu}_{\alpha}:=\hat{\mu}\left(\alpha u, \varrho_{\alpha, u}\right)$ and $v_{\alpha}(r):=\hat{v}\left(\varrho_{\alpha, u}, r\right), v_{0}^{\prime}(r):=\hat{v}\left(\varrho_{0, u}^{\prime}, r\right)$, which gives, together with (15) and (7), the estimate

$$
\left|\hat{\mu}_{\alpha}-\lambda^{4 / 3} \mu^{*}\right| \leqq c^{* 1 / 3} \alpha^{1 / 3} u^{1 / 2} .
$$

Consequently, as $v_{\alpha}(r)<0$, we get

$$
\mu_{\alpha}(r)>\hat{\mu}_{\alpha} \geqq \lambda^{4 / 3} \mu^{*}-c_{1} \alpha^{1 / 3} \text { for } 0 \leqq r \leqq R(\alpha),
$$

where $0<c_{1}<\infty$ depends on $u$ but not on $\alpha$. On the other hand, it is clear from the foregoing and the concavity of $r \mu_{\alpha}(r)$, that there exist similar constants $0<c_{2}, c_{3}, d_{3}<\infty$ with

$$
\mu_{\alpha}(r)<\left(\lambda^{4 / 3} \mu^{*}+\alpha c_{2}\right)\left(1-r^{\prime} / r\right) \text { for all } r \geqq r^{\prime}
$$

if $0 \leqq \alpha \leqq d_{3} \leqq d_{2}$, and

$$
\left|4 \pi \int_{r^{\prime}}^{R(\alpha)} \varrho_{\alpha}(r) r^{2} d r-(1-\lambda)\right| \leqq \alpha c_{3} .
$$

Putting all estimates together and using the bound (A2.4) of Appendix 2, we get the following relation for the (dimensionless) quantity

$$
\begin{gathered}
\Delta(\alpha)=\frac{4 \pi}{3} u^{3 / 2}(1-\lambda)^{-1}\left(R^{3}(\alpha)-\left(r^{\prime}\right)^{3}\right) \\
\frac{1}{5}(1-\lambda) \Delta(\alpha) \alpha^{3 / 2} \exp \left[-\frac{1}{\alpha}\left(d^{*}+\alpha^{1 / 3} c_{1} / u\right)\right] \leqq 1-\lambda+\alpha c_{3}
\end{gathered}
$$

and

$$
1-\lambda-\alpha c_{3} \leqq 2 \alpha^{1 / 2} u^{3 / 2}\left(r^{\prime}\right)^{3}+(1-\lambda) \Delta(\alpha) \exp \left[-\frac{1}{\alpha}\left(d^{*}-\alpha^{1 / 3} c_{2} / u\right)\left(1-\alpha^{1 / 3}\right)\right]
$$

if $0<\alpha \leqq d_{2}$, where $d^{*}$ equals $-\mu^{*} / u^{*}>0$. [The contribution of the interval $r^{\prime} \leqq r \leqq \alpha^{-1 / 3} r^{\prime}$ to the integral of the right hand side of (22) has been estimated separately.]

Introducing appropriate new constants $0<c, d<\infty, c_{3} \leqq c$ which are, as the previous ones, $\alpha$-independent functions of $u, r^{\prime}$ we end up with

$$
\frac{1}{\alpha}\left(d^{*}-\alpha^{1 / 3} c\right) \leqq \log \Delta(\alpha) \leqq \frac{1}{\alpha}\left(d^{*}+\alpha^{1 / 3} c\right)
$$

if $0<\alpha \leqq d$. This relation can be inverted to give

$$
d^{*} \xi\left(1-\xi^{1 / 3} e^{*}\right) \leqq \alpha_{-}(R) \leqq \alpha_{+}(R) \leqq d^{*} \xi\left(1+\xi^{1 / 3} e^{*}\right) \leqq 2 d^{*} \xi \leqq d^{* 3} / c^{3},
$$


where

$$
\xi^{-1}:=\log \Delta^{\prime}(R):=\log \left[\frac{4 \pi}{3} u^{3 / 2}(1-\lambda)^{-1}\left(R^{3}-\left(r^{\prime}\right)^{3}\right)\right]
$$

and $e^{*}:=2^{1 / 3} c\left(d^{*}\right)^{-2 / 3}$, if $\log \Delta^{\prime}(R) \geqq 2 c^{3}\left(d^{*}\right)^{-2}+2 d^{*} / d+1$, or equivalently $R \geqq R^{\prime}(u)$.

From the estimate

$$
s_{F}(\tau, v) \leqq 2+\frac{3}{2} \log \tau-\log v
$$

[where $s_{F}(\tau, v)$ is the entropy per particle of a free fermi gas with energy per particle $\tau$ and density $v$, see (A1.3) in Appendix 1] together with (8), we deduce the bound

$$
\sup \left\{4 \pi \int_{0}^{r^{\prime}} \sigma_{\alpha}(r) r^{2} d r \mid \alpha \in J(R)\right\}<h\left(u, r^{\prime}\right):=6+5\left(\sqrt{u} r^{\prime}\right)^{3}
$$

with $\sigma_{\alpha}(r):=\sigma\left(\alpha u, \varrho_{\alpha, u}(r)\right)$, using the estimate $\int_{\Lambda} \varrho(x) \log \left(a^{2} / \varrho(x)\right) d^{3} x<a^{2} .|\Lambda|$ valid for all $0 \leqq \varrho \in \mathscr{L}^{1}(\Lambda)$. [In fact, the contribution of the interval $0 \leqq r \leqq r^{\prime}$ to $\hat{s}\left(\alpha u, \varrho_{\alpha}\right)$ vanishes in the limit $R \rightarrow \infty$.] On the other hand,

$$
\sup \left\{4 \pi \int_{r^{\prime}}^{R} \sigma_{\alpha}(r) r^{2} d r \mid \alpha \in J(R)\right\}<5+\left(1-\lambda+\alpha_{+}(R) c\right) \log \Delta^{\prime}(R)
$$

if $R \geqq R^{\prime}$, using the inequality $-\int_{A} \varrho(x) \log \varrho(x) d^{3} x \leqq\|\varrho\|_{1} \log \left(|\Lambda| /\|\varrho\|_{1}\right)$ valid for all $0 \leqq \varrho \in \mathscr{L}^{1}(\Lambda)$. Combining (26) and (27) we get

$$
s^{\prime}(u, R)<h^{\prime}\left(u, r^{\prime}\right)+(1-\lambda(u)) \log \Delta^{\prime}(R)
$$

with $h^{\prime}\left(u, r^{\prime}\right)=h\left(u, r^{\prime}\right)+2 c d^{*}+5>0$, and consequently

$$
\limsup _{R \rightarrow \infty}\left(\log \left|S_{R}\right|\right)^{-1} s^{\prime}(u, R) \leqq 1-\left(u / u^{*}\right)^{3 / 4} .
$$

To calculate $\varepsilon^{\prime}(u)$, we conclude from (7) and (15), that

$$
\left|\hat{w}\left(\varrho_{\alpha}\right)-\hat{w}\left(\varrho_{0}^{\prime}\right)\right|=\frac{1}{2}\left|\int_{\mathbb{R}^{3}}\left(\varrho_{\alpha}+\varrho_{0}^{\prime}\right)\left(v_{\alpha}-v_{0}^{\prime}\right) d^{3} x\right|<\left(\alpha c^{*}\right)^{1 / 3} \sqrt{u} ;
$$

by (24) we have $\alpha_{+}(R)<2 d^{*}\left(\log \Delta^{\prime}(R)\right)^{-1}$, and consequently $\lim _{R \rightarrow \infty} \hat{w}\left(\varrho_{\alpha(R), u}\right)=\hat{w}\left(\varrho_{0, u}^{\prime}\right)$ for all $\alpha(R) \in J(R, u)$. On the other hand, using

$$
0 \leqq \theta\left(\alpha u,-\mu_{\alpha}(r)\right)-\theta\left(0,-\mu_{\alpha}(r)\right) \leqq 2 \alpha u^{5 / 2}
$$

[see (A2.6) in Appendix 2] and $0 \leqq \varrho_{\alpha}^{-1}(r) \theta\left(\alpha u,-\mu_{\alpha}(r)\right) \leqq 4 \alpha u$ if $r \geqq r^{\prime}$ and $0<\alpha \leqq d$ [see (8)] we get the inequality $\left|\hat{\tau}\left(\alpha u, \varrho_{\alpha}\right)-\hat{\tau}\left(0, \varrho_{0}^{\prime}\right)\right| \leqq \alpha u\left(\left(3 r^{\prime} \sqrt{u}\right)^{3}\left(1+c^{*}\right)+4\right)$ where the bound $\left|\theta\left(0,-\mu_{\alpha}\right)-\theta\left(0,-\mu_{0}\right)\right| \leqq 4 u\left\|\varrho_{\alpha}^{\prime}-\varrho_{0}^{\prime}\right\|_{\infty}$ follows from the convexity of $v \rightarrow \theta(0,-\mu(0, v))=\frac{3}{5} v(3 v)^{2 / 3}$ and the bound $\left\|\varrho_{\alpha, u}^{\prime}\right\|_{\infty} \leqq 2 u^{3 / 2}$ for all $0 \leqq \alpha \leqq 1$. Therefore,

$$
\varepsilon^{\prime}(u)=\lim _{R \rightarrow \infty} \hat{\varepsilon}\left(\alpha(R) u, \varrho_{\alpha(R)}\right)=\hat{\varepsilon}\left(0, \varrho_{0}^{\prime}\right)=\left(u / u^{*}\right)^{7 / 4} \varepsilon^{*}>\varepsilon
$$


for all $\alpha(R) \in J(R)$, using the scaling property $\hat{\varepsilon}\left(0, \varrho_{0, u}^{\prime}\right)=\left(u / u^{*}\right)^{7 / 4} \cdot \hat{\varepsilon}\left(0, \varrho^{*}\right)$ of the ground state T.F. energy functional. As $\varepsilon^{\prime}(u)>\varepsilon$ by the original assumption about $u$, we finally get

$$
g(\varepsilon) \leqq \inf \left\{1-\left(u / u^{*}\right)^{3 / 4} \mid\left(u / u^{*}\right)^{7 / 4} \varepsilon^{*}>\varepsilon\right\}=1-\left(\varepsilon / \varepsilon^{*}\right)^{3 / 7} .
$$

Together with (24) and the relation $\mu^{*}=\frac{7}{3} \varepsilon^{*}$ (which is a consequence of the scaling property of the ground state T.F. equation, see [6]), this proves vi) and the first part of ii) in the theorem.

\section{A Variational Lower Bound for the Rescaled Entropy}

As the entropy $s(\varepsilon, \Lambda)$ is given by (a thermodynamic limit $N \rightarrow \infty$ of) the maximum over all configurations with energies in a given interval, we get a lower bound for $s(\varepsilon, \Lambda)$ by considering a special set of orthonormal wave functions

$$
\psi_{N, i}:\left\langle\psi_{N, i}, \psi_{N, j}\right\rangle=\delta_{i j} \text { and }\left\langle\psi_{N, i}, H_{N, \Lambda}^{\sim} \psi_{N, i}\right\rangle \leqq \varepsilon N .
$$

For a given $\varepsilon$ with $\varepsilon^{*}<\varepsilon<0$, we choose a $\delta$ with $0<\delta<\left(2\left|\varepsilon^{*}\right|\right)^{-1}\left(\varepsilon-\varepsilon^{*}\right)$, define $\lambda(\delta):=\left(\varepsilon / \varepsilon^{*}+\delta\right)^{3 / 7}<1$ and fix an $r^{\prime}>\lambda(\delta)^{-1 / 3} R^{*}$. In the sequel, we shall consider product wave functions $\psi_{N, i}$ where $M$ particles are in the ground state of $H_{M, S_{r^{\prime}}}$ and $N-M$ particles are in an eigenstate (with energy $E_{i} \leqq\left|\varepsilon^{*}\right| \delta N$ ) of the free (rescaled) Hamiltonian

$$
N^{-2 / 3} 2^{-2 / 3} \pi^{-4 / 3} \sum_{j=1}^{N-M}\left(-\Delta_{j}\right) \quad \text { on } \quad \mathscr{H}\left(S_{R} \backslash S_{r^{\prime}}\right), \quad R>r^{\prime}
$$

with Dirichlet boundary conditions on both boundaries of $\Lambda_{R}:=S_{R} \backslash S_{r^{\prime}}$ (the two spheres are supposed to have the same center). Putting $M=\min \{n \in \mathbb{N} n / N \geqq \lambda(2 \delta)\}$ gives $M \leqq N$ and $0<\lambda(\delta)<\lambda(2 \delta)=\lim _{N \rightarrow \infty} M / N<1$. If $N$ is sufficiently large, we get therefore

$$
\left\langle\psi_{N, i}, H_{N, S_{R}}^{\sim} \psi_{N, i}\right\rangle<\varepsilon^{*}\left(\lambda(\delta)^{7 / 3}-\delta\right) N<\varepsilon N
$$

for all $i \in \mathbb{N}$ with $E_{i} \leqq\left|\varepsilon^{*}\right| \delta N$, because the gravitational interaction is purely attractive. Taking the limit $N \rightarrow \infty$ gives (for sufficiently large $R$ ) the lower bound

$(1-\lambda)\left[1+\frac{3}{2} \log \left((1-\lambda)^{-1}\left|\varepsilon^{*}\right| \delta\right)+\log c_{R}+3 \log \left(1-2 \sqrt{1-\lambda}\left(\left|\varepsilon^{*}\right| \delta\right)^{-1 / 2} c_{R}^{-1 / 3}\right)\right]<s\left(\varepsilon, \Lambda_{R}\right)$

[with $\lambda:=\lambda(2 \delta)$ and $\left.c_{R}:=(1-\lambda)^{-1}\left|\Lambda_{R}\right|\right]$ where we have used the estimate (A1.3) in Appendix 1

$$
1+\frac{3}{2} \log \tau-\log v+3 \log \left(1-2 v^{1 / 3} \tau^{-1 / 2}\right)<s_{F}(\tau, v),
$$

$s_{F}(\tau, v)$ being the entropy per particle of a free fermi gas with energy per particle $=\tau$ and density $v$. Choosing an $R$-dependent $\delta=\delta_{R}$ with $\lim _{R \rightarrow \infty} \delta_{R}=0,1 / \delta_{R}=o\left(c_{R}^{2 / 3}\right)$ and $\log \delta_{R}=o\left(\log c_{R}\right)$ for $R \rightarrow \infty$ [which holds for every $\delta_{R}=\operatorname{const}\left(\log c_{R}\right)^{a}, a<0$ ], we 
have $\lim _{R \rightarrow \infty} \lambda\left(2 \delta_{R}\right)=\left(\varepsilon / \varepsilon^{*}\right)^{3 / 7}$ and the monotonicity of $s(\varepsilon, \Lambda)$ in $\Lambda$ gives the lower bound

$$
\lim _{n \rightarrow \infty} \inf \left(\log \left|\Lambda_{n}\right|\right)^{-1} S\left(\varepsilon, \Lambda_{n}\right) \geqq 1-\left(\varepsilon / \varepsilon^{*}\right)^{3 / 7} \Theta(-\varepsilon)
$$

for any sequence $\left(\Lambda_{n}\right)_{n \in \mathbb{N}}$ of bounded open regions with $\lim _{n \rightarrow \infty}\left|\Lambda_{n}\right|^{-1}=0$ and $\lim _{n \rightarrow \infty}\left(\log \left|\Lambda_{n}\right|\right)^{-1} \log R_{-}\left(\Lambda_{n}\right)=1 / 3$. (For $\varepsilon>0$, we simply have to put $\lambda \equiv 0=r^{\prime}$, to replace $\Lambda_{R}$ by $S_{R}$ and to set $\delta_{R} \equiv \varepsilon / \varepsilon^{*}$.) By continuous extension to $\varepsilon=\varepsilon^{*}$ and $\varepsilon=0$, this proves, together with (33), statement ii) in the theorem.

As the inequality (24) shows that the convergence

$$
\lim _{R \rightarrow \infty} T_{ \pm}^{\prime}\left(s\left(\varepsilon, S_{R}\right), S_{R}\right) \log \left|S_{R}\right|=\left(-\frac{7}{3} \varepsilon^{*}\right)\left(\varepsilon / \varepsilon^{*}\right)^{4 / 7}
$$

is uniform on compact subsets of $\left(\varepsilon^{*}, 0\right), T^{\prime \prime}\left(s\left(\varepsilon, S_{R}\right), S_{R}\right) \log \left|S_{R}\right|$ also tends to the above limit and therefore the length of the interval $I\left(s\left(\varepsilon, S_{R}\right), S_{R}\right)$ vanishes in the limit $R \rightarrow \infty$ for $\varepsilon^{*}<\varepsilon<0$. This generalizes the notation of convergence for the microcanonical free energy and proves, together with ii) the first part of iii) in the theorem, the remaining part being a consequence of v) and (35).

\section{Appendix 1}

It has been shown in [3], that for $S_{F}(E, N, \Lambda)$ the entropy of $N$ free fermions at energy $E$ in a volume $\Lambda$, the following estimate holds

$$
S_{M B}^{\prime}(E, N, \Lambda) \leqq S_{F}(E, N, \Lambda) \leqq S_{M B}(E, N, \Lambda),
$$

where

$$
S_{M B}(E, N, \Lambda):=\log \frac{1}{N !} \operatorname{Tr}_{M B} \Theta\left(E-H_{N, \Lambda}\right)
$$

and

$$
S_{M B}^{\prime}(E, N, \Lambda):=\log \frac{1}{N !} \cdot \operatorname{Tr}_{M B}\left[\Theta\left(E-H_{N, \Lambda}\right) \prod_{i=1}^{N} \Theta\left(H_{i, \Lambda}-e_{0}\right)\right] .
$$

Here $\operatorname{Tr}_{M B}$ means trace over the Maxwell-Boltzmann-Hilbert space, i.e. the (full) tensor product $\mathscr{L}^{2}\left(\Lambda^{N}\right)$ of the one particle spaces. $H_{N, \Lambda}=\sum_{i=1}^{N} H_{i, \Lambda}$ is the (free) Hamiltonian and $e_{0}$ the lowest eigenvalue of $H_{N, A}$. Taking the (usual) thermodynamic limit we get

$$
\begin{gathered}
\frac{3}{2} \log \left(\left(3 h^{2}\right)^{-1} 4 \pi m \tau\right)+3 \log \left(1-\left(\frac{\tau_{F}}{\tau}\right)^{1 / 2}\right)-\log v+\frac{5}{2}<s_{F}(\tau, v) \\
<\frac{3}{2} \log \left(\left(3 h^{2}\right)^{-1} 4 \pi m \tau\right)-\log v+\frac{5}{2},
\end{gathered}
$$


where the Fermi energy $\tau_{F}$ for (spinless) fermions with density $v$ is given by $\tau_{F}=\frac{h^{2}}{8 m}\left(\frac{6}{\pi} v\right)^{2 / 3}$. Using our numerical choice of units, we finally get

$$
1+\frac{3}{2} \log \tau+3 \log \left(1-2 v^{1 / 3} \tau^{-1 / 2}\right)-\log v<s_{F}(\tau, v)<2+\frac{3}{2} \log \tau-\log v .
$$

\section{Appendix 2}

From the definition of $\theta(T,-\mu)$ and $v(T,-\mu)$ given explicitly in Sect. 1, we can easily obtain the bounds

$$
\frac{1}{10} \mu^{5 / 2}+\frac{1}{2}(\ln 2) T \mu^{3 / 2}+\frac{1}{2} T^{5 / 2}<\theta(T,-\mu)<\frac{1}{5} \mu^{5 / 2}+2^{-1 / 2}(\ln 2) T \mu^{3 / 2}+\frac{9}{10} T^{5 / 2}
$$

respectively

$$
\frac{1}{6} \mu^{3 / 2}+2^{-3 / 2}(\ln 2) T \mu^{1 / 2}+\frac{1}{5} T^{3 / 2}<v(T,-\mu)<\frac{1}{3} \mu^{3 / 2}+\frac{1}{2}(\ln 2) T \mu^{1 / 2}+\frac{2}{5} T^{3 / 2}
$$

if $\mu \geqq 0$ and

$$
\frac{3}{16} \sqrt{\pi} T^{5 / 2} \exp (\mu / T)<\theta(T,-\mu)<\frac{3}{8} \sqrt{\pi} T^{5 / 2} \exp (\mu / T)
$$

respectively

$$
\frac{\sqrt{\pi}}{8} T^{3 / 2} \exp (\mu / T)<v(T,-\mu)<\frac{\sqrt{\pi}}{4} T^{3 / 2} \exp (\mu / T)
$$

for $\mu \leqq 0$.

This leads to the estimates

$$
\frac{1}{3} T<\frac{1}{v} \theta(T,-\mu)<4(\mu \Theta(\mu)+T),
$$

where $\mu=\mu(T, v)$ and

$$
\begin{gathered}
\theta_{F}(0, v)=\frac{3}{5} v(3 v)^{2 / 3}, \quad \mu(0, v)=(3 v)^{2 / 3} \\
\theta_{F}(0, v) \leqq \theta_{F}(T, v) \leqq \theta_{F}(0, v) \Theta(\mu)+T\left(\mu^{3 / 2} \Theta(\mu)+T^{3 / 2}\right),
\end{gathered}
$$

where $\theta_{F}(T, v):=\theta(T,-\mu(T, v)$ and $\mu \equiv \mu(T, v)$.

\section{Appendix 3}

Decomposing the Coulomb potential into a short range and a long range part leads to the estimate

$$
\begin{aligned}
\|\hat{v}(\varrho)\|_{\infty} & \leqq \inf \left\{\frac{1}{2} R^{2}\|\varrho\|_{\infty}+(4 \pi R)^{-1}\|\varrho\|_{1} \mid R>0\right\} \\
& =\frac{3}{2}(4 \pi)^{-2 / 3}\|\varrho\|_{\infty}^{1 / 3}\|\varrho\|_{1}^{2 / 3} .
\end{aligned}
$$


For an arbitrary $\varrho \in \mathscr{F}^{\prime}(T, \Lambda),(\mathrm{A} 2.2)$ and (A2.4) in Appendix 2 gives the bounds $\mu:=\hat{\mu}(T, \varrho)<3|\Lambda|^{-2 / 3}$ (for all $T \geqq 0$ ) and $\mu<-T$ (for $T \geqq 6|\Lambda|^{-2 / 3}$ ). Defining $u:=\hat{u}(T, \varrho)$ and $v:=\hat{\mu}(T, \varrho)-u=\inf \{\hat{v}(\varrho, x) \mid x \in \Lambda\}$ for an arbitrary $\varrho \in \mathscr{F}^{\prime}(T, \Lambda)$ we get, in the case $u \geqq 0$, from (A3.1) and (A2.2): $\varrho(x)<(u+T)^{3 / 2}$. Consequently, $u+T<u-\mu=|v|<(u+T)^{1 / 2}$ if $T \geqq 6|\Lambda|^{-2 / 3}$ and $u-3|\Lambda|^{-2 / 3}<|v|<\left(u+6|\Lambda|^{-2 / 3}\right)^{1 / 2}$ if $T \leqq 6|\Lambda|^{-2 / 3}$. This leads to the estimates

$$
|v|<1, \quad u<1-T
$$

(if $u \geqq 0$ and $6|\Lambda|^{-2 / 3} \leqq T<1-u$ ) and

$$
|v|<1+3|\Lambda|^{-1 / 3}, \quad u<1+3|\Lambda|^{-1 / 3}+3|\Lambda|^{-2 / 3}
$$

(if $u \geqq 0$ and $T \leqq 6|\Lambda|^{-2 / 3}$ ).

In the case of $u \leqq 0,(\mathrm{~A} 2.4)$ and (A3.1) give

$$
|v|<\sqrt{T} \exp \left(-\frac{1}{3 T}|u|\right)
$$

and

$$
\frac{1}{2} \sqrt{T} \exp \left(-\frac{1}{3 T}|\mu|\right)|\Lambda|^{1 / 3} \leqq 1
$$

Introducing in (A3.4) the upper bound for $\sqrt{T}$ given by (A3.5) and using $|v|=|\mu|-|u|$ if $u \leqq 0$ we get

$$
|v|<\sup \left\{\min \left(\sqrt{T}, 2|\Lambda|^{-1 / 3} \exp \left(T^{-1 / 2}\right)\right) \mid T \geqq 0\right\}<1+6|\Lambda|^{-1 / 3} .
$$

Combining all estimates, this leads to the bound

$$
\|\hat{v}(\varrho)\|_{\infty}<1+6|\Lambda|^{-1 / 3} \text { for all } \varrho \in \mathscr{F}^{\prime}(T, \Lambda) .
$$

From (A2.2), (A2.4), and (A3.6) we conclude that

$$
u<4+T(3-\ln |\Lambda|)
$$

if $T \geqq 6|\Lambda|^{-2 / 3}$, so that, for every $T>0, \hat{u}(T, \varrho) \leqq 0$ for all $\varrho \in \mathscr{F}^{\prime}(T, \Lambda)$ if $|\Lambda|$ is sufficiently large.

Acknowledgements. It is a pleasure to thank Prof. W. Thirring for his permanent interest and encouragement and Drs. B. Baumgartner, H. Grosse, and H. Narnhofer for many fruitful discussions. I have profited a great deal from the private communication of some new results by Dr. Baumgartner.

\section{References}

1. Auchmuty, J., Beals, R.: Arch. Ration Mech. Anal. 43, 255-271 (1971)

2. Baumgartner, B.: Commun. Math. Phys. 48, 207-213 (1976)

3. Baumgartner, B.: Commun. Math. Phys. 75, 25-41 (1980)

4. Hertel, P., Narnhofer, H., Thirring, W.: Commun. Math. Phys. 28, 159-176 (1972)

5. Hertel, P., Thirring, W.: Commun. Math. Phys. 24, 22-36 (1971) 
6. Hertel, P., Thirring, W.: Thermodynamic instability of a system of gravitating fermions. In: Quanten und Felder. Dürr, H.P. (ed.). Braunschweig: Vieweg 1971

7. Lieb, E., Simon, B.: Adv. Math. 23, 22-116 (1977)

8. Messer, J.: Z. Phys. B 33, 313-316 (1979)

9. Ruelle, D.: Statistical mechanics, rigorous results. New York, Amsterdam: Benjamin 1969

Communicated by E. Lieb

Received April 16, 1980

Note added in proof. The existence of a phase transition in the canonical ensemble (finite configuration space) has been shown rigorously in the mean time in Messer, J.: Existence of a phase transition in a system of gravitating fermions. University of Leuven. Preprint KUL-TF-80/17, Leuven, Belgium. 